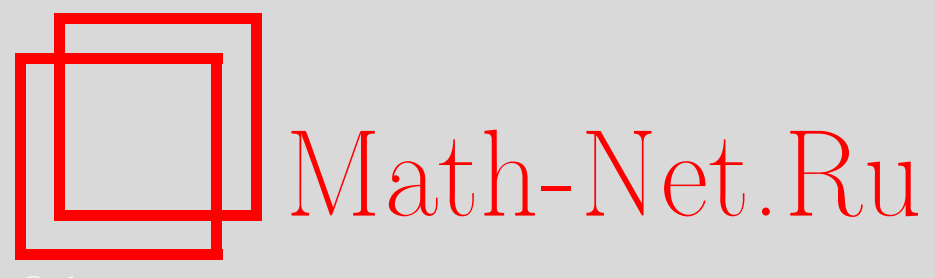

С. С. Марченков, Булева сводимость, Дискрет. матем., 2003, том 15, выпуск 3, 40-53

DOI: https://doi.org/10.4213/dm204

Использование Общероссийского математического портала Math-Net.Ru подразумевает, что вы прочитали и согласны с пользовательским соглашением http://www.mathnet.ru/rus/agreement

Параметры загрузки:

IP : 54.84 .234 .179

26 апреля 2023 г., $17: 50: 09$ 
УдК 519.716

\section{Булева сводимость}

(c) 2003 г. С. С. Марченков

На множестве всех бесконечных двоичных последовательностей определяется оператор булевой сводимости - вариант оператора конечно-автоматной сводимости, когда рассматриваются автоматы с несколькими входами и одним состоянием. Каждое множество $Q$ булевых функций, содержащее селекторную функцию и замкнутое относительно операции суперпозиции специального вида, определяет $Q$-сводимость и $Q$-степени - множества $Q$-эквивалентных последовательностей. В работе исследуются свойства частично упорядоченного множества $\mathscr{L}_{Q}$ всех $Q$-степеней: наличие максимальных, минимальных и наибольшего элементов, сушествование бесконечных цепей и антищепей, существование верхних граней.

Работа выполнена при поддержке Российского фонда фундаментальных исследований, проект 03-01-00783.

\section{1. Введение}

В теории алгоритмов в вопросах классификации множеств важная роль отводится понятиям алгоритмической сводимости и степени неразрешимости. Алгоритмическая сводимость и порождаемые ею степени вводятся по следующей схеме.

Пусть $N=\{0,1,2, \ldots\}$. Фиксируется некоторый класс $O$ эффективных операторов, заданных на булеане $\mathscr{B}(N)$. Пусть $X, Y$ - подмножества $N$. Говорят, что оператор $O$ сводит множество $X$ к множеству $Y$, если $X=O(Y)$. Множество $X$ О-сводимо к множеству $Y$ (обозначение $X \leqslant 0 Y$ ), если существует такой оператор $O$ из класса $\mathcal{O}$, который сводит $X$ к $Y$.

Обычно класс 0 содержит тождественный оператор и замкнут относительно операции композиции. Поэтому отношение $\mathcal{O}$-сводимости определяет на булеане $\mathscr{B}(N)$ предпорядок. Множества $X, Y$ называются 0 -эквивалентными, если $X \leqslant 0 \quad Y$ и $Y \leqslant 0 X$. Класс $\mathcal{O}$-эквивалентных множеств называется $\mathcal{O}$-степенью. Отношение $\leqslant 0$ индуцирует на множестве $\mathscr{L}_{\mathcal{O}}$ всех $\mathcal{O}$-степеней частичный порядок. Для наиболее употребительных алгоритмических сводимостей (e-, $T$-, $t t$ - и $m$-сводимости) соответствующее частично упорядоченное множество $\mathscr{L}_{\odot}$ образует верхнюю полурешетку с нулем (см. [1]).

В рамках приведенной выше общей схемы можно определять многие другие виды алгоритмической сводимости и соответствующие им степени. В 1974 г. Г. Рейна [2] предложил рассматривать в качестве операторов сводимости операторы конечно-автоматных преобразований. Не вдаваясь в детали определения конечно-автоматной сводимости, отметим, что она оказалась весьма своеобразной сводимостью, во многом отличающейся от известных алгоритмических сводимостей (см. [2-6]). Добавим еще, что при рассмотрении 
конечно-автоматной сводимости вместо множеств удобно пользоваться характеристическими функщиями или, что эквивалентно, бесконечными двоичными последовательностями.

Естественным шагом на пути определения “самых простых" операторов алгоритмической сводимости является рассмотрение конечно-автоматных операторов, осуществляемых автоматами с несколькими входами и одним состоянием. В этом случае автомат можно представить одной булевой функцией. Поэтому соответствующая сводимость в работе названа булевой сводимостью.

Цель настоящей работы - дать точное определение булевой сводимости, установить ее важнейшие свойства, выявить структуру частично упорядоченного множества булевых степеней и рассмотреть примеры булевой сводимости для наиболее интересных множеств булевых функций.

\section{2. Основные понятия}

Будем рассматривать бесконечные двоичные последовательности, составленные из нулей и единиц. Если $\alpha$ - такая последовательность, то ее элементы обозначаем через $\alpha_{0}, \alpha_{1}, \alpha_{2}, \ldots$ Таким образом, $\alpha=\alpha_{0} \alpha_{1} \alpha_{2} \ldots$, где $\alpha_{i} \in\{0,1\}$ при $i \geqslant 0$. Если $i \in N$, то через $(\alpha)_{i}$ обозначаем последовательность $\alpha_{i} \alpha_{i+1} \ldots$

Пусть $f\left(x_{1}, \ldots, x_{n}\right)-$ булева функция, $\alpha=\alpha_{0} \alpha_{1} \ldots, \beta=\beta_{0} \beta_{1} \ldots-$ бесконечные двоичные последовательности. Говорим, что функция $f$ сводит последовательность $\alpha$ к последовательности $\beta$, если существуют такие числа $k, l \in N$, что для любого $i \in N$ выполняется условие

$$
\alpha_{k+i}=f\left(\beta_{l+i}, \ldots, \beta_{l+i+n-1}\right) .
$$

В случае, когда $k=l=0$, говорим, что функция $f$ стандартно сводит последовательность $\alpha$ к последовательности $\beta$. Очевидно, что если функция $f$ сводит последовательность $\alpha$ к последовательности $\beta$ и выполняется соотношение (1), то функция $f$ стандартно сводит последовательность $(\alpha)_{k}$ к последовательности $(\beta)_{l}$.

Пусть $Q-$ множество булевых функций. Говорим, что последовательность $\alpha Q$-сводится к последовательности $\beta$ (обозначение $\alpha \leqslant Q \beta$ ), если множество $Q$ содержит такую функцию $f$, которая сводит $\alpha$ к $\beta$. Последовательности $\alpha, \beta$ называем $Q$-эквивалентными, если $\alpha \leqslant Q \beta$ и $\beta \leqslant Q \alpha$. Совокупность всех последовательностей, $Q$-эквивалентных последовательности $\alpha$, образует $Q$-степень, содержащую последовательность $\alpha$, которую мы обозначаем через $[\alpha]_{Q}$.

Пусть $\mathscr{L}_{Q}$ есть множество всех $Q$-степеней. Отношение $\leqslant Q$ переносим с последовательностей на $Q$-степени: если $a, b-Q$-степени, то полагаем $a \leqslant Q b$, если $\alpha \leqslant Q \beta$ для некоторых последовательностей $\alpha \in a$ и $\beta \in b$.

Мы хотим, чтобы отношение $\leqslant Q$ являлось частичным порядком на множестве $\mathscr{L}_{Q}$. Нетрудно видеть, что для этого отношение $\leqslant Q$, рассматриваемое на множестве последовательностей, должно обладать свойствами рефлексивности и транзитивности. Чтобы обеспечить рефлексивность отношения $\leqslant Q$, потребуем вхождение в множество $Q$ какойлибо селекторной функции, то есть функщии $e_{j}^{n}, 1 \leqslant j \leqslant n, n \geqslant 1$, удовлетворяющей тождеству

$$
e_{j}^{n}\left(x_{1}, \ldots, x_{j}, \ldots, x_{n}\right)=x_{j} .
$$

Действительно, если $e_{j}^{n} \in Q$, то для любой последовательности $\alpha$ функция $e_{j}^{n}$ сводит $\alpha$ 
к $\alpha$ (в качестве значений параметров $k, l$ в соответствующей формуле (1) следует взять $j-1$ и 0$)$ и, таким образом, $\alpha \leqslant Q \alpha$.

Выполнение для отношения $\leqslant Q$ свойства транзитивности приводит к замкнутости множества $Q$ относительно операции суперпозиции специального вида, которую мы будем называть $r$-суперпозицией. Говорят, что функщия $h\left(x_{1}, \ldots, x_{m+n-1}\right)$ получается из функший $f\left(x_{1}, \ldots, x_{n}\right)$ и $g\left(x_{1}, \ldots, x_{m}\right)$ с помощью операции $r$-суперпозиции, если

$$
h\left(x_{1}, \ldots, x_{m+n-1}\right)=f\left(g\left(x_{1}, \ldots, x_{m}\right), g\left(x_{2}, \ldots, x_{m+1}\right), \ldots, g\left(x_{n}, \ldots, x_{m+n-1}\right)\right) .
$$

Множество булевых функций, замкнутое относительно операции $r$-суперпозиции, назовем $r$-замкнутым.

Убедимся, что для $r$-замкнутого множества $Q$ отношение $\leqslant Q$ транзитивно на множестве последовательностей. Пусть функции $f\left(x_{1}, \ldots, x_{n}\right)$ и $g\left(x_{1}, \ldots, x_{m}\right)$ сводят соответственно последовательность $\alpha$ к последовательности $\beta$ и последовательность $\beta$ к последовательности $\gamma$. Пусть далее для функции $f$, некоторых $k, l$ и всех $i$ выполняется равенство (1), а для функции $g$, некоторых $p, q$ и всех $i$ равенство

$$
\beta_{p+i}=g\left(\gamma_{q+i}, \ldots, \gamma_{q+i+m-1}\right) \text {. }
$$

Если функция $h$ определена равенством (2), то из соотношений (1) и (3) следует, что для любых $i$ выполняется равенство

$$
\alpha_{s+i}=h\left(\gamma_{s+l+q-k-p+i}, \ldots, \gamma_{s+l+q-k-p+i+m+n-2}\right),
$$

где $s$ выбрано так, что $s \geqslant \max (k, p+k-l)$. Значит, функция $h$ сводит последовательность $\alpha$ к последовательности $\gamma$ и, таким образом, $\alpha \leqslant Q \gamma$.

Отметим, что $r$-замкнутое множество, вообще говоря, не является замкнутым относительно обычной операции суперпозиции. Это видно на примере $r$-замкнутого множества

$$
\left\{a_{1} x_{1} \oplus \ldots \oplus a_{n-1} x_{n-1} \oplus x_{n}, n=1,2, \ldots\right\} \text {. }
$$

В дальнейшем мы будем преимущественно рассматривать такие множества $Q$ булевых функций, которые $r$-замкнуты и содержат селекторную функцию. Для таких множеств $Q$ множество $\mathscr{L}_{Q} Q$-степеней частично упорядочено отношением $\leqslant Q$.

\section{3. Некоторые свойства булевой сводимости}

Предложение 1. Пусть $Q-r$-замкнутое множество булевых функций, содержащее селекторную функцию, $\alpha=\alpha_{0} \alpha_{1} \ldots$ - бесконечная двоччная последовательность. Тогда число двоччных последовательностей, $Q$-сводячихся к последовательности $\alpha$, не более чем счетно, а $Q$-степень $[\alpha]_{Q}$ содержит все последовательности вида

$$
\beta_{0} \ldots \beta_{p} \alpha_{q} \alpha_{q+1} \ldots
$$

где $\beta_{0} \ldots \beta_{p}-$ произвольная конечная двоччная последовательность (возможно пустая) $u q \in N$.

Доказательство. Первая часть утверждения следует из того, что каждый из параметров $f, k, l$, участвующих в определении $Q$-сводимости, принимает значения из не более чем счетного множества. 
Пусть в множество $Q$ входит селекторная функция $e_{j}^{n}$. Тогда функщия $e_{j}^{n}$ будет сводить последовательность (4) к последовательности $\alpha$, если в качестве значений параметров $k$, $l$ в определении сводимости выбрать $p+j$ и $q$. Обратно, последовательность $\alpha$ сводится к последовательности (4) функцией $e_{j}^{n}$, если в качестве значений параметров $k, l$ выбрать $q+j-1$ и $p+1$.

Следствие 1. Для любого $r$-замкнутого множества $Q$ булевых функций, содержащего селекторную функиию, любая $Q$-степень состочт из счетного числа различных последовательностей. Частично упорядоченное множество $\mathscr{L}_{Q}$ континуально и не имеет наибольшего элемента.

Предложение 2. Пусть $\beta$ - периодическая последовательность периода р и булева функчия $f\left(x_{1}, \ldots, x_{n}\right)$ сводит последовательность $\alpha \kappa$ последовательности $\beta$. Тогда $\alpha-$ также периодическая последовательность периода $p$.

Доказательство. Пусть для некоторых $k, l$ и всех $i$ выполняется соотношение (1). Согласно условию, найдется такое $j_{0}$, что для любого $j \geqslant j_{0}$ имеет место равенство $\beta_{j}=\beta_{p+j}$. Тогда на основании (1) для любого $i$ такого, что $l+i \geqslant j$, будет справедливо равенство $\alpha_{k+i}=\alpha_{p+k+i}$. Таким образом, $\alpha$ - периодическая последовательность периода $p$.

Следствие 2. Пусть $Q-r$-замкнутое множество булевых функиий, содержашее селекторную функчию, $\alpha$ - бесконечная периодическая последовательность. Тогда начальный сегмент

$$
\left\{a: a \in \mathscr{L}_{Q}, a \leqslant Q[\alpha]_{Q}\right\}
$$

частично упорядоченного множества $\mathscr{L}_{Q}$, определяемый элементом $[\alpha]_{Q}$, конечен.

Будем обозначать число элементов конечного множества $A$ через $|A|$.

Предложение 3. Для любой булевой функции $f\left(x_{1}, \ldots, x_{n}\right)$ существует такая периодическая последовательность $\alpha$, что функчия $f$ стандартно сводит последовательность $\alpha$ не более чем $\kappa 2^{n-1}$ двоичным последовательностям.

Доказательство. Пусть $i \in\{0,1\}$ и $f^{-1}(i)$ - полный прообраз $i$ при отображении $f$. Обозначим через $A_{0}$ то из множеств $f^{-1}(0), f^{-1}(1)$, которое содержит меньшее число элементов (при равенстве числа элементов полагаем $A_{0}=f^{-1}(0)$ ). Очевидно, что $\left|A_{0}\right| \leqslant 2^{n-1}$. Если $A_{0}=f^{-1}(i)$, то пусть $\alpha_{0}=i$. При $A_{0}=\varnothing$ полагаем

$$
\alpha_{1}=\alpha_{2}=\ldots=\alpha_{0}
$$

и тем самым завершаем определение последовательности $\alpha$.

Пусть $A_{0} \neq \varnothing$. Обозначим через $A_{1}$ то из двух множеств

$$
\left\{\left(a_{1}, a_{2}, \ldots, a_{n+1}\right):\left(a_{1}, \ldots, a_{n+1}\right) \in A_{0} \times\{0,1\}, f\left(a_{2}, \ldots, a_{n+1}\right)=i\right\},
$$

которое содержит меньшее число элементов (при равенстве числа элементов в множествах (5) в качестве $A_{1}$ выбираем множество, отвечающее значению $i=0$ ). Полагаем $\alpha_{1}=i$, если для данного $i$ множество $A_{1}$ совпадает с множеством (5). Очевидно, что $A_{1} \subseteq\{0,1\}^{n+1}$ и $\left|A_{1}\right| \leqslant\left|A_{0}\right|$.

Продолжая по индукции, предположим, что множество $A_{j}$ уже определено и $A_{j} \subseteq\{0,1\}^{n+j}$. Если $A_{j}=\varnothing$, то завершаем определение последовательности $\alpha$, полагая

$$
\alpha_{j+1}=\alpha_{j+2}=\ldots=\alpha_{j}
$$


Если же $A_{j} \neq \varnothing$, то обозначим через $A_{j+1}$ то из двух множеств

$$
\left\{\left(a_{1}, a_{2}, \ldots, a_{n+j+1}\right):\left(a_{1}, \ldots, a_{n+j+1}\right) \in A_{j} \times\{0,1\}, f\left(a_{j+2}, \ldots, a_{n+j+1}\right)=i\right\},
$$

которое содержит меньшее число элементов (при равенстве числа элементов выбираем множество (6) для $i=0$ ). Полагаем $\alpha_{j+1}=i$, если для данного $i$ множество $A_{j+1}$ совпадает с множеством (6).

Из построения следует, что либо для некоторого $j$ получаем $A_{j}=\varnothing$, и тогда $\alpha$ есть периодическая последовательность

$$
\alpha_{0} \ldots \alpha_{j-1} \alpha_{j} \alpha_{j} \alpha_{j} \ldots
$$

либо все множества $A_{0}, A_{1}, \ldots$ непусты и тогда

$$
\left|A_{0}\right| \geqslant\left|A_{1}\right| \geqslant\left|A_{2}\right| \geqslant \ldots
$$

В последнем случае положим

$$
m=\lim _{i \rightarrow \infty}\left|A_{i}\right|
$$

Если в процессе построения реализуется случай $A_{j}=\varnothing$, то по определению множества $A_{j}$ и значения $\alpha_{j}$ функция $f$ не может стандартно сводить последовательность $\alpha$ ни к какой двоичной последовательности.

Предположим, что все множества $A_{j}$ непусты. Поскольку при любом $j$ любой набор из $A_{j+1}$ получается из подходящего набора множества $A_{j}$ добавлением последнего элемента, последовательность множеств $A_{0}, A_{1}, \ldots$ в пределе определяет $m$ бесконечных двоичных последовательностей $\beta^{1}, \ldots, \beta^{m}$. Из построения следует также, что последовательность $\alpha$ стандартно сводится функцией $f$ только к одной из последовательностей $\beta^{1}, \ldots, \beta^{m}$.

Остается убедиться, что последовательность $\alpha$ периодическая. В самом деле, число множеств вида

$$
\left\{\left(a_{j+2}, \ldots, a_{j+n}\right):\left(a_{j+2}, \ldots, a_{j+n}\right) \text { есть конец некоторого набора из } A_{j}\right\}
$$

не превосходит величины $2^{2^{n-1}}$. Если для некоторых $j_{1}, j_{2}$ соответствующие множества (7) совпадают и при этом $\left|A_{j_{1}}\right|=\left|A_{j_{2}}\right|=m$, то, как вытекает из построения, будут совпадать множества (7), определенные для величин $j_{1}+1$ и $j_{2}+1$. Это обеспечивает периодичность последовательности $\alpha$. Предложение доказано.

Обозначим последовательности $00 \ldots, 11 \ldots$ через 0 и 1.

Предложение 4. Для любого $r$-замкнутого множества $Q$ булевых функций, содержащего селекторную функцию, хотя бы одна из $Q$-степеней $[0]_{Q},[1]_{Q}$ минимальна в $\mathscr{L}_{Q}$.

Доказательство. Рассмотрим последовательность 0. Если каждая функция множества $Q$ сохраняет константу 0 , то любая последовательность $\alpha, Q$-сводящаяся к последовательности 0, содержит, очевидно, лишь конечное число единиц. Пользуясь предложением 1 , заключаем, что в этом случае последовательности 0 и $\alpha Q$-эквивалентны и, следовательно, $Q$-степень $[0]_{Q}$ минимальна в $\mathscr{L}_{Q}$.

Предположим, что в множестве $Q$ имеется функция $f$, не сохраняющая 0. Тогда функция $f$ сводит к последовательности 0 любую последовательность $\beta$, которая содержит конечное число нулей. В силу предложения 1 любая такая последовательность $\beta$ 
$Q$-эквивалентна последовательности 1. Далее повторяем приведенные выше рассуждения для последовательности 1. Именно, если все функции множества $Q$ сохраняют константу 1 , то минимальной является $Q$-степень $[1]_{Q}$. В противном случае последовательности 0 и $1 Q$-эквивалентны и определяют (одну) минимальную $Q$-степень.

Предложение 5. Пусть $Q-r$-замкнутое множество булевых функций, содержащее селекторную функцию. Тогда $Q$-степени $[0]_{Q},[1]_{Q}$ являются различными минимальными элементами в $\mathscr{L}_{Q}$ в том и только том случае, когда все функции множества $Q$ сохраняют константы 0 и 1 .

Доказательство. Пусть $Q$-степени $[0]_{Q},[1]_{Q}$ суть различные минимальные элементы в $\mathscr{L}_{Q}$. Тогда, в частности, последовательность 1 не может $Q$-сводиться к последовательности 0. Отсюда вытекает (см., например, доказательство предложения 4), что все функции множества $Q$ сохраняют константу 0 . Аналогично устанавливаем, что все функции множества $Q$ сохраняют константу 1 .

Обратно, пусть все функции множества $Q$ сохраняют константы 0 и 1 . Теми же рассуждениями, что и в доказательстве предложения 4 , показываем, что $Q$-степени $[0]_{Q},[1]_{Q}$ минимальны в $\mathscr{L}_{Q}$. Кроме того, очевидно, что эти $Q$-степени различны.

Предложение 6. Пусть множество $Q$ булевых функций $r$-замкнуто и содержст селекторную функиию. Тогда множество $\mathscr{L}_{Q}$ имеет наименьший элемент а в том и только том случае, когда выполняется одно из следующих условий:

(1) $0 \in Q$ и все функиии множества $Q$ сохраняют константу 0 (в этом случае $0 \in a$ $u \mathbf{1} \notin a)$;

(2) $1 \in Q$ и все функиии множества $Q$ сохраняют константу 1 (в этом случае $1 \in a$ $u \mathbf{0} \notin a)$;

(3) $\{0,1\} \subset Q$ (в этом случае $\{0,1\} \subset a)$.

Доказательство. Пусть $a-$ наименьший элемент множества $\mathscr{L}_{Q}$. Согласно предложению $4, Q$-степень $a$ содержит хотя бы одну из последовательностей 0,1 . Предположим, что $0 \in a$. Если $1 \notin a$, то множество $Q$ не может содержать функщию, не сохраняющую константу 0 , так как иначе последовательность 1 сводилась бы этой функцией к последовательности 0 и в силу минимальности $Q$-степени $a$ последняя содержала бы последовательность 1. Итак, все функции множества $Q$ сохраняют константу 0 .

Покажем, что $0 \in Q$. Предположим противное. Тогда для произвольной функщии $f\left(x_{1}, \ldots, x_{n}\right)$ из $Q$ найдется такой набор $\left(b_{1}, \ldots, b_{n}\right)$, что $f\left(b_{1}, \ldots, b_{n}\right)=1$. Рассмотрим такую последовательность $\alpha$, которая для любой функции $f\left(x_{1}, \ldots, x_{n}\right)$ из $Q$ содержит бесконечное число соответствующих подпоследовательностей $b_{1} \ldots b_{n}$. Понятно, что в силу этого условия любая последовательность $\beta$, которая $Q$-сводится к последовательности $\alpha$, будет содержать бесконечное число единиц. Иными словами, в этом случае последовательность 0 не может $Q$-сводиться к последовательности $\alpha$. Это противоречит тому, что $Q$-степень $[0]_{Q}$ является наименьшей в $\mathscr{L}_{Q}$.

Аналогично рассматривается случай, когда $1 \in a$ и $0 \notin a$.

Обратно, легко проверяется, что выполнение любого из условий 1-3 приводит к существованию в $\mathscr{L}_{Q}$ наименьшего элемента. 


\section{4. Верхние грани и антицепи}

Теорема 1. Для любого множества $Q$ булевых функций найдутся такие последовательности $\alpha, \beta$, что ни для одной последовательности $\gamma$ не могут одновременно выполняться соотношения $\alpha \leqslant Q \gamma$ и $\beta \leqslant Q \gamma$.

Доказательство. Будем предполагать, что функции множества $Q$ перенумерованы и $f_{i}$ - функция из $Q$ с номером $i$. Зафиксируем также нумерацию множества $N^{3}$, в которой каждая тройка чисел имеет бесконечное количество номеров. Последовательности $\alpha, \beta$ будем определять с помощью пошаговой процедуры, задавая на каждом шаге конечное число элементов $\alpha$ и $\beta$. Считаем, что до шага 0 ни один элемент последовательностей $\alpha$, $\beta$ не определен.

Шаг $2 t$. Пусть до шага $2 t$ уже определены значения $\alpha_{0}, \ldots, \alpha_{k_{2 t-1}}$ и $\beta_{0}, \ldots, \beta_{k_{2 t-1}}$, и пусть $t$ есть номер тройки $(p, q, r)$. Будем предполагать, что функции $f_{p}, f_{q}$ зависят от $n$ переменных.

Используя функцию $f_{p}$, так же, как в доказательстве предложения 3 , разворачиваем процесс порождения элементов периодической последовательности

$$
\alpha_{k_{2 t-1}+1} \alpha_{k_{2 t-1}+2} \ldots
$$

и последовательностей $\delta^{1}, \ldots, \delta^{m}$ с таким расчетом, чтобы последовательность $\alpha_{k_{2 t-1}+1} \alpha_{k_{2 t-1}+2} \ldots$ стандартно сводилась функцией $f_{p}$ лишь к одной из последовательностей $\delta^{1}, \ldots, \delta^{m}$. Если для некоторого $s$ после определения значений

$$
\alpha_{k_{2 t-1}+1}, \ldots, \alpha_{k_{2 t-1}+s}
$$

выяснится, что $m=0$, то завершаем шаг $2 t$, добавляя к уже определенным элементам $\alpha_{0}, \ldots, \alpha_{k_{2 t-1}}$ новые элементы $\alpha_{k_{2 t-1}+1}, \ldots, \alpha_{k_{2 t-1}+s}$. Полагаем также

$$
\beta_{k_{2 t-1}+1}=\ldots=\beta_{k_{2 t-1}+s}=0 \text {. }
$$

Таким образом, получаем, что $k_{2 t}=k_{2 t-1}+s$. Далее переходим к шагу $2 t+1$.

Пусть $m \neq 0$. Выберем число $s$ так, чтобы (см. доказательство предложения 3) множество $A_{s}$, строящееся для определения значения $\alpha_{k_{2 t-1}+s}$, состояло из $m$ элементов. Пусть, кроме того, выполняется неравенство $s \geqslant r+m-1$. Добавляем тогда к элементам $\alpha_{0}, \ldots, \alpha_{k_{2 t-1}}$ элементы $\alpha_{k_{2 t-1}+1}, \ldots, \alpha_{k_{2 t-1}+s}$, и полагаем

$$
\beta_{k_{2 t-1}+1}=\bar{f}_{q}\left(\delta_{r}^{1}, \ldots, \delta_{r+n-1}^{1}\right), \ldots, \beta_{k_{2 t-1}+m}=\bar{f}_{q}\left(\delta_{r+m-1}^{m}, \ldots, \delta_{r+m+n-2}^{m}\right)
$$

и $\beta_{k_{2 t-1}+i}=0$ при $m<i \leqslant s$. Таким образом, получаем, что $k_{2 t}=k_{2 t-1}+s$. Переходим к шагу $2 t+1$.

Шаг $2 t+1$. Пусть до шага $2 t+1$ уже определены значения $\alpha_{0}, \ldots, \alpha_{k_{2 t}}$ и $\beta_{0}, \ldots, \beta_{k_{2 t}}$, a $t$ есть номер тройки $(p, q, r)$. Проводим те же построения, что и на шаге $2 t$, заменяя $k_{2 t-1}$ на $k_{2 t}$ и поменяв местами $f_{p}, f_{q}$ и $\alpha, \beta$. Затем переходим к шагу $2 t+2$.

Покажем, что не существует такой последовательности $\gamma$, для которой одновременно $\alpha \leqslant Q \quad \gamma$ и $\beta \leqslant Q \quad \gamma$. Пусть это не так, последовательность $\gamma$ с указанными свойствами существует и булевы функции $f_{p}, f_{q}$ из $Q$ сводят последовательности $\alpha, \beta$ к последовательности $\gamma$. Тогда найдутся такие числа $a, b, c, d$, что функция $f_{p}$ стандартно сводит 
последовательность $(\alpha)_{a}$ к последовательности $(\gamma)_{b}$, а функщия $f_{q}$ стандартно сводит последовательность $(\beta)_{c}$ к последовательности $(\gamma)_{d}$. Нетрудно видеть, что в этом случае для любого $v \geqslant \max (a, c)$ найдутся такие числа $z, w$, что функии $f_{p}, f_{q}$ будут стандартно сводить последовательности $(\alpha)_{v}$ и $(\beta)_{v}$ к последовательностям $(\gamma)_{z}$ и $(\gamma)_{w}$ соответственно. В самом деле, достаточно положить $z=b+v-a$ и $w=d+v-c$.

Пусть

$$
r=|w-z|=|a+d-b-c| .
$$

Рассмотрим случай, когда $a+d-b-c \geqslant 0$. Тогда последовательность $(\gamma)_{w}$ есть конец последовательности $(\gamma)_{z}$. Выберем такой номер $t$ тройки $(p, q, r)$, чтобы выполнялось неравенство $k_{2 t-1} \geqslant \max (a, c)$. Если на шаге $2 t$ выясняется, что $m=0$, то элементы $\alpha_{k_{2 t-1}+1}, \ldots, \alpha_{k_{2 t-1}+s}$ последовательности $\alpha$ определяются в конструкции таким образом, что функщия $f_{p}$ не может стандартно сводить последовательность $(\alpha)_{k_{2 t-1}+1}$ ни к какой последовательности $\gamma$.

Предположим, что на шаге $2 t$ значение соответствующей величины $m$ не равно 0 . Тогда значения $\alpha_{k_{2 t-1}+1}, \ldots, \alpha_{k_{2 t-1}+s}$ определяются в конструкщии так, что функщия $f_{p}$ может стандартно сводить последовательность $(\alpha)_{k_{2 t-1}+1}$ толькс к одной из последовательностей $\delta^{1}, \ldots, \delta^{m}$. Вместе с тем равенства (8) показывают, что функция $f_{q}$ не может стандартно сводить последовательность $(\beta)_{k_{2 t-1}+1}$ к последовательности $\left(\delta^{1}\right)_{r}$, последовательность $(\beta)_{k_{2 t-1}+2}$ к последовательности $\left(\delta^{2}\right)_{r+1}, \ldots$, последовательность $(\beta)_{k_{2 t-1}+m}$ к последовательности $\left(\delta^{m}\right)_{r+m-1}$. Получаем противоречие с утверждением о существовании последовательностей вида $(\gamma)_{z}$ и $(\gamma)_{w}$, где $w-z=r$.

В случае $a+d-b-c<0$ рассматриваем подходящий шаг вида $2 t+1$, где $t$ есть номер тройки $(p, q, r)$.

Теорема доказана.

Следствие 3. Для любого $r$-замкнутого множества $Q$ булевых функиий, содержащего селекторную функцию, частично упорядоченное множество $\mathscr{L}_{Q}$ не является верхней полурешеткой.

Замечание 1. Полученное следствие указывает на значительное отличие булевой сводимости от известных алгоритмических сводимостей $(e-, T-, t t-, m-$ и конечно-автоматной сводимости), для которых соответствующее множество $\mathscr{L}_{Q}$ образует верхнюю полурешетку.

Подмножество $A$ частично упорядоченного множество $\mathscr{L}$ называется антицепью, если любые два элемента множества $A$ не сравнимы в смысле отношения частичного порядка на $\mathscr{L}$.

Теорема 2. Пусть $Q-r$-замкнутое множество булевых функций, содержашее селекторную функцию, а - элемент множества $\mathscr{L}_{Q}$, не являюшийся наименьшим. Тогда в $\mathscr{L}_{Q}$ существует континуальная античепь, включающая элемент а.

Доказательство. Поскольку элемент $a$ не является наименьшим, найдется такой элемент $b_{1}$, что $a \nless Q b_{1}$. Согласно следствию из предложения 1 элемент $a$ не может быть наибольшим в $\mathscr{L}_{Q}$. Значит, существует такой элемент $b_{2}$, что $b_{2} \nless a$. Пусть $\alpha, \beta^{1}, \beta^{2}-$ последовательности, входящие соответственно в $Q$-степени $a, b_{1}, b_{2}$.

Мы определим континуальное семейство $\left\{\gamma_{\xi}\right\}$ двоичных последовательностей таким образом, что множество $Q$-степеней

$$
\bigcup_{\xi}\left[\gamma_{\xi}\right]_{Q} \cup\{a\}
$$


будет являться антицепью в $\mathscr{L}_{Q}$. Последовательности $\gamma_{\xi}$ определяются ниже с помощью пошаговой процедуры. На каждом шаге $t$ этой процедуры образуется множество $D_{t}$ конечных двоичных последовательностей, которое состоит из $2^{t}$ последовательностей, причем ни одна из них не является началом другой. Вместе с тем каждая последовательность из $D_{t}$ служит началом ровно двух последовательностей из $D_{t+1}$. В пределе множества $D_{t}$ определят искомое континуальное семейство $\left\{\gamma_{\xi}\right\}$.

Будем предполагать, что множество $Q$ перенумеровано и $f_{m}-$ функщия из $Q$ с номером $m$. Зафиксируем, кроме того, некоторую нумерацию множества $N^{3}$.

Шаг 0. Образуем множество $D_{0}$ из последовательностей 0 и 1.

Шаг $t+1$. Пусть $t$ есть номер тройки $(k, l, m)$, функция $f_{m}$ из $Q$ зависит от $n$ переменных и уже определено множество $D_{t}=\left\{d_{1}, \ldots, d_{2^{t}}\right\}$ конечных последовательностей, каждая из которых не является началом другой. Поскольку $a \nless{ }_{Q} b_{1}$, функция $f_{m}$ не может сводить последовательность $\alpha$ ни к одной из последовательностей $d_{1} \beta^{1}, \ldots, d_{2^{t}} \beta^{1}$. Значит, для любого $j, 1 \leqslant j \leqslant 2^{t}$, найдется такое число $i_{j}$, что

$$
\alpha_{k+i_{j}} \neq f_{m}\left(\delta_{l+i_{j}}, \ldots, \delta_{l+i_{l}+n-1}\right),
$$

где $\delta_{l+i_{j}} \ldots \delta_{l+i_{j}+n-1}$ - начало последовательности $\left(d_{j} \beta^{1}\right)_{l+i_{j}}$. Если величина $l+i_{j}+n-1$ больше длины последовательности $d_{j}$, то определяем $d_{j}^{1}$ равной началу последовательности $d_{j} \beta^{1}$ длины $l+i_{j}+n$. В противном случае полагаем $d_{j}^{1}=d_{j}$.

Выполняя переход от последовательностей $d_{1}, \ldots, d_{2^{t}}$ к последовательностям $d_{1}^{1}, \ldots, d_{2^{t}}^{1}$, мы обеспечиваем стандартную несводимость с помощью функции $f_{m}$ последовательности $(\alpha)_{k+i_{j}}$ к любой последовательности $(\delta)_{l+i_{j}}$, где началом $\delta$ служит последовательность $d_{j}^{1}$.

Этот технический прием с небольшими изменениями мы применяем на шаге $t+1$ еще $2^{t}$ раз. Именно, рассматриваем последовательность $d_{1}^{1} \beta^{2}$. Она $Q$-эквивалентна последовательности $\beta^{2}$ и потому в силу выбора $\beta^{2}$ последовательность $d_{1}^{1} \beta^{2}$ не может сводиться функцией $f_{m}$ ни к одной из последовательностей $\alpha, d_{2}^{1} \alpha, \ldots, d_{2^{t}}^{1} \alpha$ (которые все $Q$-эквивалентны последовательности $\alpha$ ). Обозначив через $\delta^{2}, \ldots, \delta^{2^{t}}$ последовательности $d_{2}^{1} \alpha, \ldots, d_{2^{t}}^{1} \alpha$, заключаем, что можно выбрать такое число $p$, что каждая из последовательностей

$$
\begin{gathered}
f_{m}\left(\alpha_{l}, \ldots, \alpha_{l+n-1}\right) f_{m}\left(\alpha_{l+1}, \ldots, \alpha_{l+n}\right) \ldots f_{m}\left(\alpha_{l+p}, \ldots, \alpha_{l+p+n-1}\right) \\
f_{m}\left(\delta_{l}^{2}, \ldots, \delta_{l+n-1}^{2}\right) f_{m}\left(\delta_{l+1}^{2}, \ldots, \delta_{l+n}^{2}\right) \ldots f_{m}\left(\delta_{l+p}^{2}, \ldots, \delta_{l+p+n-1}^{2}\right) \\
\ldots \\
f_{m}\left(\delta_{l}^{2^{t}}, \ldots, \delta_{l+n-1}^{2^{t}}\right) f_{m}\left(\delta_{l+1}^{2^{t}}, \ldots, \delta_{l+n}^{2^{t}}\right) \ldots f_{m}\left(\delta_{l+p}^{2^{t}}, \ldots, \delta_{l+p+n-1}^{2^{t}}\right)
\end{gathered}
$$

будет отличаться от начала последовательности $\left(d_{1}^{1} \beta^{2}\right)_{k}$ длины $p+1$. Считая $p$ достаточно большим, полагаем $d_{1}^{2}$ равным началу последовательности $d_{1}^{1} \beta^{2}$ длины $k+p$, a $d_{2}^{2}, \ldots, d_{2^{t}}^{2}$ - началам соответственно последовательностей $d_{2}^{1} \alpha, \ldots, d_{2^{t}}^{1} \alpha$ длины $l+p+n$.

Выполняя эту часть шага $t+1$, мы обеспечиваем стандартную несводимость с помощью функции $f_{m}$ любой последовательности $\left(\delta^{1}\right)_{k}$, для которой $d_{1}^{2}$ служит началом последовательности $\delta^{1}$, к последовательности $(\alpha)_{l}$, а также к любым последовательностям $\left(\delta^{2}\right)_{l}, \ldots,\left(\delta^{2^{t}}\right)_{l}$, для которых $d_{2}^{2}, \ldots, d_{2^{t}}^{2}$ служат началами последовательностей $\delta^{2}, \ldots, \delta^{2^{t}}$. 
Далее рассматриваем последовательность $d_{2}^{2}$ и поступаем так же, как при рассмотрении последовательности $d_{1}^{1}$, используя уже построенные последовательности $d_{1}^{2}, d_{3}^{2}, \ldots, d_{2^{t}}^{2}$. В результате образуем последовательности $d_{1}^{3}, d_{2}^{3}, \ldots, d_{2^{t}}^{3}$ и так далее. После того, как будут получены последовательности $d_{1}^{2^{t}+1}, \ldots, d_{2^{t}}^{2^{t}+1}$, завершаем шаг $t+1$, полагая

$$
D_{t+1}=\left\{d_{1}^{2^{t}+1} 0, d_{1}^{2^{t}+1} 1, \ldots, d_{2^{t}}^{2^{t}+1} 0, d_{2^{t}}^{2^{t}+1} 1\right\} .
$$

Из построения следует, что семейство последовательностей $\left\{\gamma_{\xi}\right\}$, образованное из множеств $D_{t}$ переходом к пределу, будет задавать в $\mathscr{L}_{Q}$ континуальную антицепь, удовлетворяющую условиям теоремы.

\section{5. Примеры}

(1a) $Q_{1}=\left\{e_{1}^{1}(x)\right\}$. Очевидно, что если последовательность $\alpha$ сводится к последовательности $\beta$ функцией $e_{1}^{1}$, то $\alpha$ имеет вид $\alpha_{0} \ldots \alpha_{k-1}(\beta)_{k}$. Значит, последовательность $\alpha Q_{1}$-эквивалентна последовательности $\beta$. Таким образом, каждая последовательность $\beta$ порождает в $\mathscr{L}_{Q_{1}}$ минимальный элемент, а множество $\mathscr{L}_{Q_{1}}$ представляет собой континуальную антицепь.

(1b) $Q_{2}=\left\{0, e_{1}^{1}(x)\right\}$. В соответствии с предложением 6 , множество $\mathscr{L}_{Q_{2}}$ имеет наименьший элемент $[0]_{Q_{2}}$. Оставшаяся часть $\mathscr{L}_{Q_{2}}$, так же, как и в примере 1а, представляет собой континуальную антицепь.

(1c) $Q_{3}=\left\{0,1, e_{1}^{1}(x)\right\}$. Здесь предложение 6 дает в множестве $\mathscr{L}_{Q_{3}}$ наименьший элемент, порожденный последовательностями 0 и 1. Как и в примере $1 \mathrm{~b}$, над наименьшим элементом в $\mathscr{L}_{Q_{3}}$ располагается континуальная антицепь.

(1d) $Q_{4}=\left\{e_{1}^{1}(x), \bar{e}_{1}^{-1}(x)\right\}$. Нетрудно видеть, что в этом случае для любой последовательности $\alpha Q_{4}$-степень $[\alpha]_{Q_{4}}$ получается объединением $Q_{1}$-степеней $[\alpha]_{Q_{1}}$ и $[\bar{\alpha}]_{Q_{1}}$, где $\bar{\alpha}=\bar{\alpha}_{0} \bar{\alpha}_{1} \ldots$ В остальном этот пример близок к примеру $1 \mathrm{a}:$ частично упорядоченное множество $\mathscr{L}_{Q_{4}}$ является континуальной антицепью.

(1e) $Q_{5}=\left\{0,1, e_{1}^{1}(x), \bar{e}_{1}^{\mathrm{i}}(x)\right\}$. Здесь получаем аналог примера 1с: множество $\mathscr{L}_{Q_{5}}$ имеет наименьший элемент, над которым расположена континуальная антицепь.

$$
Q_{6}=\left\{x_{1} \vee \ldots \vee x_{n} ; n=1,2, \ldots\right\}
$$

Предложение 5 гарантирует существование в множестве $\mathscr{L}_{Q_{6}}$ двух минимальных элементов, определяемых последовательностями 0 и 1. Покажем, что других минимальных элементов в $\mathscr{L}_{Q_{6}}$ нет.

Предположим, напротив, что $\alpha$ - последовательность с бесконечным числом нулей и единиц и $Q_{6}$-степень $[\alpha]_{Q_{6}}$ минимальна в $\mathscr{L}_{Q_{6}}$, и пусть последовательность $\beta$ сводится к последовательности $\alpha$ функцией $x_{1} \vee \ldots \vee x_{c}$. В силу минимальности $Q_{6}$-степени $[\alpha]_{Q_{6}}$ последовательность $\alpha$ будет сводиться к последовательности $\beta$ некоторой функцией $x_{1} \vee \ldots \vee x_{d}$. Пользуясь транзитивностью сводимости и свойствами дизъюнкции, заключаем, что последовательность $\alpha$ сводится к самой себе функцией $x_{1} \vee \ldots \vee x_{c+d-1}$. Случай $c+d-1=1$ тривиален: последовательность $\beta$ сводится к последовательности $\alpha$ селекторной функцией. Покажем, что случай $c+d-1 \geqslant 2$ невозможен. 
В целях упрошения обозначений положим $n=c+d-1$, и пусть $n \geqslant 2$. Считаем, что функщия $x_{1} \vee \ldots \vee x_{n}$ стандартно сводит последовательность $(\alpha)_{k}$ к последовательности $(\alpha)_{l}$.

Пусть $k>l$. Возьмем такое $i$, что $\alpha_{l+i}=1$. Пользуясь стандартной сводимостью последовательности $(\alpha)_{k}$ к последовательности $(\alpha)_{l}$, заключаем, что должно быть, по крайней мере, $\alpha_{k+i}=1$. Поскольку $k>l$, воспользуемся еще раз стандартной сводимостью последовательности $(\alpha)_{k}$ к $(\alpha)_{l}$ и получим, что $\alpha_{k+(k-l)+i}=1$. Повторяя это рассуждение, приходим к выводу, что для любого $j \geqslant 0$ имеет место равенство $\alpha_{k+j}(k-l)+i=1$. Однако в этом случае последовательность 1 сводится к последовательности $\alpha$ функцией $x_{1} \vee \ldots \vee x_{k-l}$, что противоречит минимальности $Q_{6}$-степени $[\alpha]_{Q_{6}}$ и неравенству $[1]_{Q_{6}} \neq[\alpha]_{Q_{6}}$.

Пусть $k \leqslant l$. Рассмотрим некоторую конечную подпоследовательность из нулей, входящую в последовательность $\alpha$ после разряда $\alpha_{k-1}$. Иными словами, пусть $i$ и $p$ таковы, что

$$
\alpha_{k+i}=\alpha_{k+i+1}=\ldots=\alpha_{k+i+p}=0 .
$$

Пользуясь стандартной сводимостью последовательности $(\alpha)_{k}$ к последовательности $(\alpha)_{l}$ функцией $x_{1} \vee \ldots \vee x_{n}$, заключаем, что справедливы равенства

$$
\alpha_{l+i}=\alpha_{l+i+1}=\ldots=\alpha_{l+i+p+n-1}=0 \text {. }
$$

Так как $k \leqslant l$, из этих равенств и стандартной сводимости $(\alpha)_{k}$ к $(\alpha)_{l}$ функщией $x_{1} \vee \ldots \vee x_{n}$ вытекает, что

$$
\alpha_{l+(l-k)+i}=\alpha_{k+(l-k)+i+1}=\ldots=\alpha_{l+(l-k)+i+p+2(n-1)}=0 .
$$

Аналогичным образом устанавливаем, что вообще для любого $j \geqslant 0$ имеют место равенства

$$
\alpha_{l+j(l-k)+i}=\alpha_{k+j(l-k)+i+1}=\ldots=\alpha_{l+j(l-k)+i+p+(j+1)(n-1)}=0 .
$$

Однако для всех достаточно больших $j$ справедливы неравенства

$$
l+j(l-k)+i \leqslant l+(j+1)(l-k)+i \leqslant l+j(l-k)+i+p+(j+1)(n-1) .
$$

Это означает, что последовательность $\alpha$ содержит лишь конечное число единиц, что невозможно по предположению. Таким образом, $\mathscr{L}_{Q_{6}}$ не содержит минимальных элементов, отличных от $[0]_{Q_{6}}$ и $[1]_{Q_{6}}$.

Нетрудно видеть, что $Q_{6}$-степень $[0]_{Q_{6}}$ является не только минимальной, но и максимальной в $\mathscr{L}_{Q_{6}}$. В самом деле, если последовательность 0 сводится к последовательности $\alpha$ функцией $x_{1} \vee \ldots \vee x_{n}$, то последовательность $\alpha$ необходимо содержит лишь конечное число единиц. Значит, последовательность $\alpha Q_{6}$-эквивалентна последовательности 0.

Множество $\mathscr{L}_{Q_{6}}$ имеет еще континуальное число максимальных элементов. Чтобы убедиться в этом, рассмотрим бесконечную последовательность $i_{0}, i_{1}, \ldots$ чисел из $N$ такую, что для любого $j \geqslant 0$ выполняется неравенство $i_{j+1}-i_{j} \geqslant 2$. Определим последовательность $\beta$ условиями

$$
\beta_{i}= \begin{cases}1, & \text { если } i \in\left\{i_{0}, i_{1}, \ldots\right\} \\ 0 & \text { в противном случае }\end{cases}
$$

Покажем, что $Q_{6}$-степень $[\beta]_{Q_{6}}$ максимальна в $\mathscr{L}_{Q_{6}}$. 
Предположим, что последовательность $\beta$ сводится к последовательности $\gamma$ функщией $x_{1} \vee \ldots \vee x_{n}$. Если $n=1$, то, очевидно, последовательность $\gamma Q_{6}$-эквивалентна последовательности $\beta$. Пусть $n \geqslant 2$. Возьмем такое достаточно большое число $j$, чтобы выполнялись условия:

$$
\begin{aligned}
\beta_{i_{j}-1} & =\gamma_{p} \vee \ldots \vee \gamma_{p+n-1}, \\
\beta_{i_{j}} & =\gamma_{p+1} \vee \ldots \vee \gamma_{p+n}=1, \\
\beta_{i_{j}+1} & =\gamma_{p+2} \vee \ldots \vee \gamma_{p+n+1} .
\end{aligned}
$$

Так как $\beta_{i_{j}}=1$, одно из чисел $\gamma_{p+1}, \ldots, \gamma_{p+n}$ равно 1. Тогда ввиду неравенства $n \geqslant 2$ будет равна 1 хотя бы одна из дизъюнкций $\gamma_{p} \vee \ldots \vee \gamma_{p+n-1}, \gamma_{p+2} \vee \ldots \vee \gamma_{p+n+1}$. Однако согласно выбору последовательности $\left\{i_{0}, i_{1}, \ldots\right\}$ должно быть $\beta_{i_{j}-1}=\beta_{i_{j}+1}=0$. Противоречие показывает, что последовательность $\beta$ не может сводиться ни к какой последовательности $\gamma$ функцией $x_{1} \vee \ldots \vee x_{n}$, где $n \geqslant 2$. Значит, элемент [ $\left.\beta\right]_{Q_{6}}$ максимален в множестве $\mathscr{L}_{Q_{6}}$.

Так как существует континуальное множество последовательностей $\left\{i_{0}, i_{1}, \ldots\right\}$ с указанными выше свойствами, приходим к выводу, что в $\mathscr{L}_{Q_{6}}$ существует континуальное число максимальных элементов.

$Q_{6}$-степень $[1]_{Q_{6}}$, очевидно, не является максимальной в $\mathscr{L}_{Q_{6}}$. Покажем, что в $\mathscr{L}_{Q_{6}}$ имеется еще континуальное число немаксимальных элементов. Пусть $i_{0}, i_{1}, \ldots$ - последовательность натуральных чисел, больших 1. Определим двоичную последовательность $\beta$ следующим образом. Пусть $\beta_{0}=1$. Далее полагаем равными 0 следующие $i_{0}$ разрядов последовательности $\beta$. Затем в последовательности $\beta$ помещаем два блока вида $110^{i_{1}}\left(0^{i}\right.$ обозначает последовательность, состоящую из $i$ нулей). Вообще, после последнего блока вида $1^{k} 0^{i_{k}-1}$ в последовательности $\beta$ располагаем $k+1$ блоков вида $1^{k+1} 0^{i_{k}}$.

Пусть последовательность $\gamma^{1}$ получается из последовательности $\beta$ заменой первой единицы каждого блока, состоящего целиком из единиц, нулем. Легко видеть, что последовательность $\beta$ сводится к последовательности $\gamma^{1}$ функщией $x_{1} \vee x_{2}$. Вместе с тем последовательность $\gamma^{1}$ не может $Q_{6}$-сводиться к последовательности $\beta$, поскольку при достаточно больших $k$ идущие подряд $k$ блоков $1^{k-1}$ последовательности $\gamma^{1}$ не могут получаться из единичных блоков последовательности $\beta$ добавлением фиксированного числа единиц.

Нетрудно понять, что элемент $\left[\gamma^{1}\right] Q_{6}$ также не является максимальным в $\mathscr{L}_{Q_{6}}$. Из последовательности $\gamma^{1}$ заменой первой единицы каждого блока, состоящего из единиц, нулем получаем последовательность $\gamma^{2}$ такую, что $\gamma^{1}$ сводится к $\gamma^{2}$ функцией $x_{1} \vee x_{2}$, но $\gamma^{2}$ не входит в $Q_{6}$-степень $\left[\gamma^{1}\right]_{6}$. Этот процесс позволяет определить над элементом $[\beta]_{Q_{6}}$ бесконечную возрастающую цепь.

(2b), (2c), (2d) В этих примерах

$$
\begin{aligned}
& Q_{7}=\left\{0, x_{1} \vee \ldots \vee x_{n} ; n=1,2, \ldots\right\}, \\
& Q_{8}=\left\{1, x_{1} \vee \ldots \vee x_{n} ; n=1,2, \ldots\right\}, \\
& Q_{9}=\left\{0,1, x_{1} \vee \ldots \vee x_{n} ; n=1,2, \ldots\right\} .
\end{aligned}
$$

Отличия множеств $\mathscr{L}_{Q_{7}}, \mathscr{L}_{Q_{8}}, \mathscr{L}_{Q_{9}}$ от множества $\mathscr{L}_{Q_{6}}$ состоят в следующем. В множестве $\mathscr{L}_{Q_{7}}$ элемент [0] $Q_{7}$ является наименьшим, а элемент [1] $Q_{7}$ - атомом. Остальные структурные особенности множества $\mathscr{L}_{Q_{6}}$ сохраняются. В множестве $\mathscr{L}_{Q_{8}}$ элемент $[1]_{Q_{8}}$ является наименьшим, а элемент $[0]_{Q_{8}}$ - атомом и одновременно наибольшим элементом. В множестве $\mathscr{L}_{Q_{9}}$ наименьший элемент содержит обе последовательности 0 и 1. 
(3) $Q_{10}-$ множество всех булевых функщий.

Согласно предложению 6 множество $\mathscr{L}_{Q_{10}}$ имеет наименьший элемент, содержащий по-

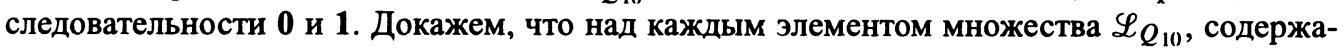
щим периодическую последовательность, существует счетная возрастающая цепь. Пусть $\alpha$ - периодическая последовательность периода $p \geqslant 2$. Без ограничения общности можно считать, что $\alpha$ - чисто периодическая последовательность. Рассмотрим периодическую последовательность $\beta^{1}$ периода $2 p$, составленную из блоков $10^{2 p-1}$. Тогда $\alpha \leqslant Q_{10} \beta^{1}$. В самом деле, последовательность $\alpha$ стандартно сводится к последовательности $\beta^{1}$ любой функцией $f\left(x_{1}, \ldots, x_{2 p}\right)$, для которой выполняются соотношения

$$
\begin{aligned}
f(1,0, \ldots, 0) & =\alpha_{0}, \quad f(0, \ldots, 0,1)=\alpha_{1}, \\
f(0, \ldots, 0,1,0) & =\alpha_{2}, \ldots, f(0,1,0, \ldots, 0)=\alpha_{2 p-1}
\end{aligned}
$$

Вместе с тем последовательность $\beta^{1}$ не может $Q_{10}$-сводиться к последовательности $\alpha$, поскольку в этом случае в силу предложения 2 последовательность $\beta^{1}$ имела бы период $p$.

Далее, аналогичным образом определяем периодические последовательности $\beta^{2}, \beta^{3}, \ldots$, имеющие соответственно периоды $10^{4 p-1}, 10^{8 p-1} \ldots$ Понятно, что

$$
\beta^{1} \leqslant Q_{10} \beta^{2} \leqslant Q_{10} \beta^{3} \leqslant Q_{10} \ldots
$$

однако в силу предложения 2 соотношения

$$
\beta^{2} \leqslant Q_{10} \beta^{1}, \quad \beta^{3} \leqslant Q_{10} \beta^{2}, \ldots
$$

невозможны.

Заметим, что в приведенном выше построении вместо последовательности $\beta^{1}$ с равным успехом можно взять любую последовательность с периодом $10^{d p-1}$, где $d \geqslant 3$. Поэтому на самом деле над элементом $[\alpha]_{Q_{10}}$ в множестве $\mathscr{L}_{Q_{10}}$ располагается континуальное число счетных возрастающих цепей.

Описанное выше свойство элементов множества $\mathscr{L}_{Q_{10}}$, содержащих периодические последовательности, присуще не только этим элементам. Имеется еще континуальное множество элементов, которые обладают этим свойством. Пусть $i_{0}, i_{1}, \ldots$ - последовательность чисел из $N$ такая, что

$$
2<i_{1}-i_{0}<i_{2}-i_{1}<i_{3}-i_{2}<\ldots
$$

Определим двоичную последовательность $\alpha$ условиями:

$$
\alpha_{i}= \begin{cases}1, & \text { если } i \in\left\{i_{0}, i_{1}, \ldots\right\} \\ 0 & \text { в противном случае. }\end{cases}
$$

Возьмем произвольную двоичную последовательность $\gamma$ и определим двоичную последовательность $\beta$, полагая $\beta_{i_{j}+1}=\gamma_{j}, \beta_{l}=\alpha_{l}$ для остальных значений $l$. Очевидно, что последовательность $\alpha$ сводится к последовательности $\beta$ функщией $\bar{x}_{1} x_{2}$. Поскольку существует континуальное число последовательностей $\beta$, найдется такая последовательность $\beta^{1}$, которая не $Q_{10}$-эквивалентна последовательности $\alpha$. С последовательностью $\beta_{1}$ поступаем так же, как с последовательностью $\alpha$ : для любой двоичной последовательности $\gamma$ полагаем $\beta_{i j+2}=\gamma_{j}, \beta_{l}=\beta_{l}^{l}$ для остальных значений $l$. В качестве булевой функции, сводящей последовательность $\beta^{1}$ к любой такой последовательности $\beta$, можно взять функцию $\bar{x}_{1} \bar{x}_{2} x_{3}$ и так далее. 


\section{Список литературы}

1. Роджерс Х., Теория рекурсивных функиий и эффективная вычислимость. Мир, Москва, 1972.

2. Рейна Г., Степени автоматных преобразований. Кибери. сб. (1977), 14, 95-106.

3. Gordon H. G., Complete degrees of finite-state tranformability. Information and Control (1976) 32, 169-187.

4. Байрашева В. Р., Структурные свойства автоматных преобразований. Известия вузов. Математика (1988) 7, 34-39.

5. Марченков С. С., Конечные начальные сегменты верхней полурешетки конечно-автоматных степеней. Дискретная математика (1989) 1, №3, 96-103.

6. Соловьев В. Д., Структура распределения информации в бесконечной последовательности. Дискретная математика (1996) 8, №2, 97-107.

Статья поступила 31.10.2002. 